diseño, fabricación y optimización de los dispositivos de liberación controlada con geometría de toro.

Mathematical modeling of controlled drug release from matrix-type torus-shaped vaginal devices

\section{Summary}

The design, development and use of vaginal rings have increased focus and attention in science, industry and society in general. This technology is still in its beginning and is expected to have an important role in the pharmaceutical industry in the coming years. The use of mathematical modeling as a tool to guide the development and optimization of these controlled release devices is a key factor in the progress and development of this technology.

In this regard, the development of a reliable mathematical model capable to predict the release kinetics from vaginal rings was the central goal of present study. The model developed was rigorously validated by comparison with experimental profiles obtained in our laboratory and with experimental data reported in the literature by other authors.
The use of this model allows to study the controlled release of solute from rings through computer simulations, thus avoiding the inconveniences of the in vitro and in vivo experimentation.

The model developed was successfully used to predict the release of different hormonal solutes, covering in this task, the variation of the polymer that forms the matrix of the devices, the variation of design parameters such as the initial load of solute and the dimensions of the rings, the variation of experimental parameters such as the agitation rate and the release medium, among others. The successful results obtained in this wide range of assays allow to asseverate the validity and usefulness of the model developed.

Finally, the model developed was successfully used for the optimization of an available commercial device. The use of the model allowed the study and the comparison of the system by computer simulations, which established the basis to improve an existing vaginal ring. Therefore, it can be concluded that the use of the mathematical model developed is an invaluable tool in the design, manufacture and optimization of torus-shaped controlled release devices.

\title{
Estudio de la adsorción de compuestos biorrefractarios en solución acuosa
}

\section{Pablo Danilo Húmpola}

phumpola@fbcb.unl.edu.ar

Dr. José Luis Vicente (INIFTA-CONICET-UNLP) Laboratorio de Investigación del Departamento de Química General e Inorgánica, FBCB-UNL. Instituto de Investigaciones Fisicoquímica Teóricas y Aplicadas-Área Fisi- coquímica de Superficies

Universidad Nacional de La Plata

Fecha de defensa: 11/10/2013

\section{Resumen}

En este trabajo se busca proponer nuevos puntos de vista sobre algunos de los 
problemas que surgen para tratar de describir el proceso de adsorción de adsorbatos orgánicos, en general, y de fenol, en particular, desde solución acuosa, cuando se emplean como adsorbentes carbones activados (CAs) de los más empleados comercialmente. Los numerosos estudios, realizados sobre el proceso de adsorción de fenol y sus derivados, han generado una serie de interrogantes que permanecen sin responder. Si bien se logró enumerar los factores más relevantes que afectan el proceso, no se ha podido cuantificar el peso relativo de uno frente a otro y, más aún, se desconoce cómo influye cada factor, y como compiten entre sí. Se seleccionaron dos CAs que, de acuerdo a sus fabricantes se los denominó "Norit" y "Tetrahedron", y que presentaban una estructura que evidenciaba no solo una importante presencia de "microporos" (poros cuyos diámetros son inferiores a $2 \mathrm{~nm}$ ), sino además poros de mayores dimensiones en su superficie.

En primer lugar se caracterizaron ambos carbones en forma estándar, empleando adsorción y desorción de gases simples $\left(\mathrm{N}_{2}\right.$ a $77 \mathrm{~K}$ y CO$_{2}$ a $273.2 \mathrm{~K}$. El cálculo de la superficie específica BET, mostró para el CA Norit, una mayor capacidad de adsorción.

En segundo lugar se desarrolló una metodología que permitió estudiar ambos CAs, en particular, y otros tipos de adsorbentes en general, basada en la descomposición de cada isoterma en una superposición de diversos mecanismos que se producen a medida que la presión aumenta ( se comienza a bajas presiones completando capas (Langmuir) si la superficie es suave, o el llenado de poros (Dubinin) si la superficie es heterogénea o porosa; luego sigue el llenado capa por capa (layer-by-layer) en las homogéneas, y en las heterogéneas depen- derá de la característica de los poros y la interacción entre las moléculas adsorbidas (BET m-capas o Aranovich). Para la región microporosa (bajas presiones) se observó una mayor capacidad de adsorción en el CA Norit y el modelo de Aranovich, dio un mejor ajuste en este CA, y permitió describir correctamente su comportamiento diferencial, con respecto al CA Tetradedron, en la región de altas presiones.

En tercer lugar se determinaron y analizaron en forma estándar las isotermas de adsorción de fenol en fase acuosa a tres temperaturas 293 K, 303 K y 313 K. En este caso, al igual que se hizo en forma equivalente con la fase gaseosa, se consideró primero solo el rango de bajas concentraciones (por debajo de 0,2 $\mathrm{C}_{\text {eq }}$ fenol/ (mol $\left.\mathrm{L}^{-1}\right)$ ) y luego concentraciones mayores. Para este análisis, se aplicaron isotermas como Freundlich y Sips (Langmuir-Freundlich), según los distintos rangos de concentraciones considerados. Se observó una inversión de las isotermas de adsorción, al pasar de bajas a altas concentraciones. En el CA Norit la presencia de microporos de mayor volumen, más anchos y más distribuidos, favorecen su capacidad de adsorción a bajas concentraciones. Las isotermas de adsorción son mejor descriptas con el modelo de Sips, cuando se considera todo el rango de concentraciones analizado, favoreciéndose la adsorción en el CA Tetrahedron a altas concentraciones.

En cuarto lugar se analizaron los mecanismos se ponen en juego, mayoritariamente, cuando se considera la adsorción de fenol en solución acuosa, tomando en cuenta el entorno implicado en tal proceso. Para esto se discutieron los resultados de la literatura, frente a las simulaciones numéricas y cálculos computacionales basados 
en la Teoría del Funcional de la Densidad (DFT) y dinámica molecular, que realizamos con estos sistemas. Se compararon los distintos grupos superficiales oxigenados ácidos y el efecto de cada uno de ellos en la adsorción, determinando la presencia o no de tales grupos mediante titulación selectiva de Boehm. La diferencia en el comportamiento de la adsorción de fenol en ambos CAs desde soluciones acuosas, pudo vincularse con la presencia de grupos superficiales carboxilos en el CA Norit. Los cálculos mecanocuánticos que consideran solo la interacción de la molécula de fenol con una superficie de grafeno, no mostraron diferencias, ante la presencia o no de grupos superficiales carboxilos, pero cuando se incorpora la presencia del solvente, los cálculos muestran la influencia de los grupos carboxilos en la interacción de la molécula de fenol con la superficie de grafeno. De esta manera, a diferencia de lo sugerido en la literatura, estos resultados muestran que las interacciones $\pi-\pi$ no juegan un papel determinante en el mecanismo de adsorción.

\section{Study of adsorption of compounds in aqueous solutions biorefractary}

\section{Summary}

In this paper we intend to provide new insights into some of the problems emerging when trying to describe the process of adsorption of organic adsorbates in general, and more specifically, of phenol, from aqueous solution, when the most commercially employed activated carbons (ACs) are used as adsorbents.

First, both carbons were described according to standard procedures, using adsorption and desorption of simple gases $\left(\mathrm{N}_{2}\right.$ at
$77 \mathrm{~K}$ and $\mathrm{CO}_{2}$ at $273,2 \mathrm{~K}$ ). The calculation of the BET surface area, showed for Norit AC, increased adsorption capacity.

Second, a methodology was developed where each isotherm was analyzed in terms of the superposition of different mechanisms which operates pressure increases. Higher adsorption capacity in the AC Norit and model Aranovich observed, gave a better fit in the AC, and allowed properly describe their differential behavior with respect to AC Tetradedron, region high pressure.

Third, adsorption isotherms of phenol in the liquid phase were determined and analyzed in the standard way at three temperatures $293 \mathrm{~K}, 303 \mathrm{~K}$ and $313 \mathrm{~K}$. An investment of adsorption isotherms, from low to high concentrations was observed. The presence of AC Norit micropores of larger volume, wider and more distributed, favors its adsorption capacity at low concentrations. The adsorption isotherms are best described with the Sips model, when considering the entire range of tested concentrations, favoring the adsorption at high concentrations AC Tetrahedron.

Fourth, we analyzed the mechanisms that play a part, mostly when phenol adsorption in aqueous solution is considered, taking into account the environment involved in such a process. To this end, the results of the literature review were considered together with the numerical simulations and computer calculations based on DFT and molecular dynamics that were carried out with these systems. The difference in adsorption behavior in both ACs phenols from aqueous solutions was linked to the presence of carboxyl groups on the surface Norit. The quantum mechanical calculations that consider only the interaction of the phenol molecule with a graphene sur- 
face, showed no difference in the presence or absence of surface carboxyl groups, but when it incorporates the presence of the solvent, the calculations show the influence of the groups carboxyl in the interaction with the phenol molecule graphene surface. Thus, contrary to what is suggested in the literature, these results show that $\pi-\pi$ interactions do not play a decisive role in the adsorption mechanism.

\section{Respuestas a plaguicidas y su relación con las interaccio- nes depredador-presa en anfibios (Amphibia, Anura) de agroecosistemas del Litoral Mesopotámico de Argentina}

\section{Celina María Junges}

cmjunges@fbcb.unl.edu.ar

Dra. Paola Mariela Peltzer

Dr. Rafael Carlos Lajmanovich

Laboratorio de Ecotoxicología - Escuela Superior de Sanidad

Facultad de Bioquímica y Ciencias Biológicas

Universidad Nacional del Litoral

Fecha de la defensa: 19/03/2013

\section{Resumen}

En los últimos años, se ha puesto en evidencia que la presencia ambiental de plaguicidas está relacionada con efectos indirectos en los anfibios que se manifiestan a nivel de comunidad. Una explicación posible a este fenómeno podría atribuirse a que la incorporación de estos estresores en los ecosistemas no es la suficiente para causar la muerte fisiológica de los organismos, pero si capaz afectar su funcionamiento en un contexto ecológico, efecto conocido como muerte ecológica. Entre los distintos biomarcadores que se utilizan para detectar los efectos adversos de los plaguicidas sobre los anfibios se pueden citar las respuestas etológicas y las metabólicas.

El objetivo general de esta tesis fue analizar las interacciones depredador-presa entre distintas especies de larvas de anfibios y un pez depredador del litoral mesopotámico argentino, y establecer su relación con las variaciones en las respuestas etológicas (actividad natatoria y respiración) de las presas y metabólicas (actividad de las enzimas acetilcolinestera [AChE] y butirilcolinesterasa [BChE]) de depredador y presas en presencia de concentraciones subletales de tres plaguicidas: fenitrotion ( $F$ ), glifosato (Gly) y trifloxistrobin (TFS). Para la realización de las experiencias se seleccionó a Synbranchus marmoratus como depredador y a nueve especies de larvas de anfibios como presas: Rhinella arenarum (Bufonidae), Leptodactylus latrans, L. chaquensis (Leptodactylidae), Physalaemus santafecinus, $P$. albonotatus, $P$. riograndensis (Leiuperidae), Trachycephalus typhonius, Hypsiboas pulchellus (Hylidae) y Elachistocleis bicolor (Microhylidae). Bajo condiciones contraladas de laboratorio, se llevaron a cabo las experiencias que fueron organizadas en tres etapas: la primera de ecotoxicidad, la segunda de exposición, y la tercera de ensayos de depredación y evaluación de respuestas etológicas y metabólicas.

La primera etapa consistió en evaluar la toxicidad de las larvas de anfibios frente a for- 